\title{
The protease CspB is essential for initiation of cortex hydrolysis and dipicolinic acid (DPA) release during germination of spores of Clostridium perfringens type $\mathrm{A}$ food poisoning isolates

\author{
Daniel Paredes-Sabja, ${ }^{1}$ Peter Setlow ${ }^{2}$ and Mahfuzur R. Sarker ${ }^{1,3}$
} \\ Correspondence \\ Mahfuzur R. Sarker \\ sarkerm@oregonstate.edu \\ Received 16 May 2009 \\ Revised 29 June 2009 \\ Accepted 17 July 2009

\author{
${ }^{1}$ Department of Biomedical Sciences, College of Veterinary Medicine, Oregon State University, \\ Corvallis, OR 97331, USA \\ ${ }^{2}$ Department of Molecular, Microbial and Structural Biology, University of Connecticut Health \\ Center, Farmington, CT 06030, USA \\ ${ }^{3}$ Department of Microbiology, College of Science, Oregon State University, Corvallis, OR 97331, \\ USA
}

\begin{abstract}
The genome of the Clostridium perfringens food poisoning isolate SM101 encodes a subtilisin-like protease, $\mathrm{CspB}$, upstream of the sleC gene encoding the enzyme essential for degradation of the peptidoglycan cortex during spore germination. $\mathrm{SleC}$ is an inactive pro-SleC in dormant spores that is converted to active SleC during spore germination and Csp proteases convert pro-SleC to the active enzyme in vitro. In this work, the germination and viability of spores of a $\operatorname{csp} B$ deletion mutant of strain SM101, as well as $\operatorname{csp} B$ expression, were studied. The $\operatorname{cspB}$ gene was expressed only during sporulation, and only in the mother cell compartment. $\operatorname{cs} p B$ spores were unable to germinate significantly with either a rich nutrient medium, $\mathrm{KCl}$, or a $1: 1$ chelate of $\mathrm{Ca}^{2+}$ and dipicolinic acid (DPA); the viability of these spores was $\sim 10^{4}$-fold lower than that of wild-type spores, although $\operatorname{csp} B$ and wild-type spores had similar viability on plates containing lysozyme, and $\operatorname{csp} B$ spores could not process inactive pro-SleC into active SleC during spore germination. Germination of $\operatorname{csp} B$ spores was blocked prior to DPA release and cortex hydrolysis, and germination and viability defects in these spores were complemented by an ectopic cspB. These results indicate that Csp proteases are essential to generate active SleC and allow cortex hydrolysis early in C. perfringens spore germination. However, Csp proteases likely play another role in spore germination, since $\operatorname{csp} B$ spores did not release DPA upon exposure to germinants, while sleC spores have been shown previously to release DPA, albeit slowly, upon exposure to germinants.
\end{abstract}

\section{INTRODUCTION}

Clostridium perfringens is an anaerobic, spore-forming pathogenic bacterium, and many strains cause gastrointestinal (GI) diseases in humans and animals (McClane, 2007). C. perfringens spores are metabolically dormant, resistant to many environmental insults and, once conditions are favourable, these spores can germinate, outgrow, return to vegetative growth and then release toxins and cause disease (McClane, 2007; McDonnell, 1986; ParedesSabja et al., 2008b).

Abbreviations: Ca-DPA, $\mathrm{Ca}^{2+}$-dipicolinic acid chelate; CLE, cortex-lytic enzyme; DPA, dipicolinic acid; FP, food poisoning; GUS, $\beta$-glucuronidase; $P G$, peptidoglycan.

Two supplementary tables, listing bacterial strains and plasmids and primers used in this study, and a supplementary figure, showing sequence alignment of Csp proteins of C. perfringens strains, are available with the online version of this paper.
C. perfringens spores initiate germination when they sense a variety of compounds termed germinants, which include Lasparagine, $\mathrm{KCl}$, a $1: 1$ chelate of $\mathrm{Ca}^{2+}$ and pyridine-2,6dicarboxylic acid (dipicolinic acid; DPA) (Ca-DPA) and a cationic surfactant (Paredes-Sabja et al., 2008b). In spores of Bacillus species, nutrient germinants are sensed by specific germinant receptors located in the spore inner membrane, each encoded by a tricistronic operon of the gerA family (Setlow, 2003). Although there is no intact tricistronic gerA-type operon in $C$. perfringens, the products of the bicistronic gerKAC operon are essential for spore germination with either L-asparagine or Ca-DPA, and partially required for germination with $\mathrm{KCl}$, and there is also a divergently transcribed gerKB cistron (ParedesSabja et al., 2008b). Upon binding of the germinant to its cognate receptor, DPA is released from the spore core as a $1: 1$ chelate with divalent cations, predominantly $\mathrm{Ca}^{2+}$. This event, at least in Bacillus spores, triggers the initiation 
of cortex hydrolysis through the activation of the cortexlytic enzyme (CLE) CwlJ, which is present in dormant spores as the mature, potentially active enzyme (Paidhungat et al., 2001). In C. perfringens, the CLE SleC alone is sufficient to degrade the spore peptidoglycan (PG) cortex, and Ca-DPA does not appear to directly activate cortex hydrolysis (Paredes-Sabja et al., 2008a, b, 2009a). $\mathrm{SleC}$ is present in dormant $C$. perfringens spores, although not in the mature potentially active form, but rather as an inactive zymogen, pro-SleC, which is cleaved into active SleC early in spore germination (Urakami et al., 1999). These findings suggest that signalling pathways differ in at least some aspects of germination in spores of Bacillus and Clostridium species. Ultimately, removal of the PG cortex eliminates a physical constraint, allowing the core to expand and hydrate to levels found in vegetative cells (Popham et al., 1996), thus restoring enzyme activity and metabolism and allowing spore outgrowth (Cowan et al., 2003; Setlow, 2003).

Work with C. perfringens S40 has identified three germination-specific serine proteases (Csp proteases) that belong to the subtilisin subfamily (Shimamoto et al., 2001; Siezen \& Leunissen, 1997). The majority of subtilisins are synthesized as pre-pro-enzymes, subsequently secreted into an external medium in a pro-form following the removal of the prepeptide (or signal peptide), and activated autocatalytically by cleavage of the pro-peptide (Shinde \& Inouye, 1996). Mature subtilisins possess three conserved residues (Asp, His, Ser) that form the catalytic triad of these enzymes (Siezen \& Leunissen, 1997), and the three Csp proteins in C. perfringens S40 possess this catalytic triad as well as the oxyanion binding hole (Shimamoto et al., 2001). The Csp proteins are synthesized in the mother cell compartment of the sporulating cell and subsequently translocated into the PG cortex of the developing forespore, and during this period there is processing at both the $\mathrm{N}$ and $\mathrm{C}$ termini, generating pro-SleC (Masayama et al., 2006; Shimamoto et al., 2001). In vitro studies have shown that a fraction from germinated C. perfringens S40 spore extracts containing the three Csp proteins can process pro-SleC to SleC, thus activating the enzyme for degradation of the cortex of decoated spores (Kumazawa et al., 2007; Okamura et al., 2000; Shimamoto et al., 2001). Surprisingly, genome sequencing (Myers et al., 2006; Shimizu et al., 2002) indicates that $C$. perfringens type A food poisoning (FP) isolates encode only one Csp, CspB, although non-FP isolates such as $S 40$ carry a $\operatorname{csp} A B C$ tricistronic operon encoding all three Csp proteins (Fig. 1a). However, the precise function of Csp proteins in C. perfringens spore germination is unclear. Consequently, in the current work we have constructed a $\operatorname{csp} B$ mutant of a $C$. perfringens $\mathrm{FP}$ isolate to elucidate the role of CspB in spore germination.

\section{METHODS}

Bacterial strains and plasmids. C. perfringens and plasmids used in this study are described in Supplementary Table S1.
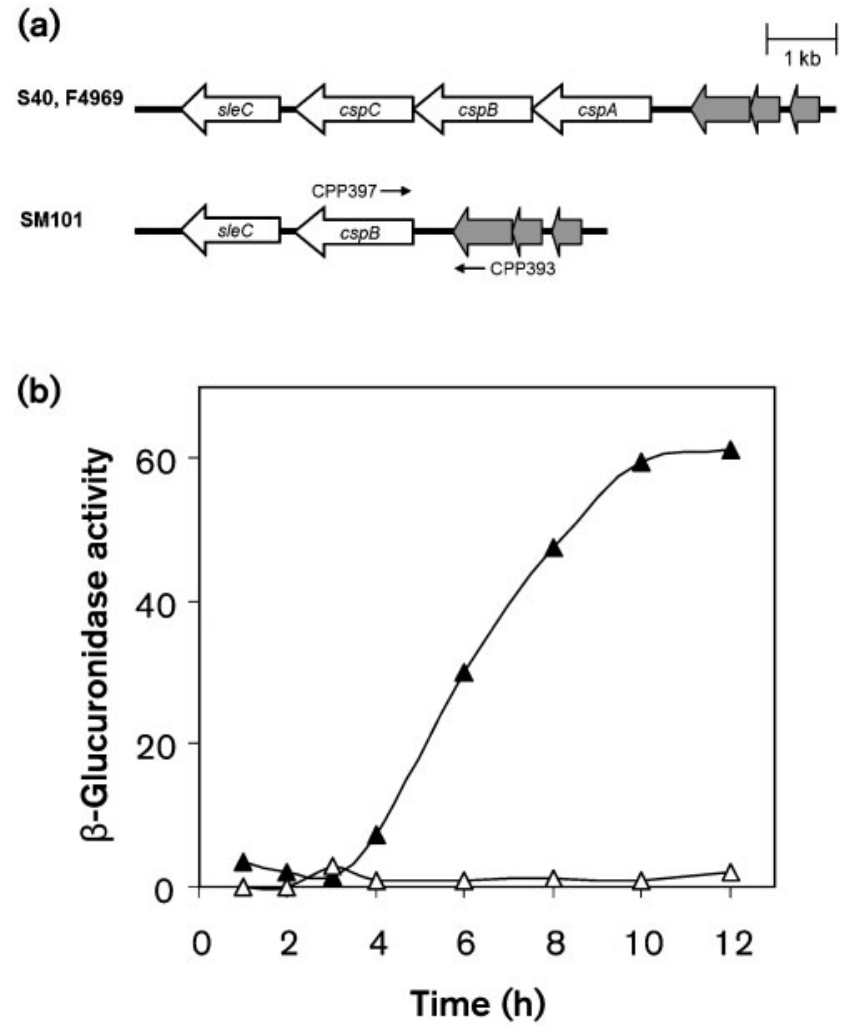

Fig. 1. (a) Genomic arrangement and (b) expression of $c s p B$ in $C$. perfringens SM101. (a) The arrangement of the csp-sleC locus in the C. perfringens FP strain SM101 and the non-FP strains F4969 and $\mathrm{S} 40$ and the location of primers used to amplify the upstream region of $\operatorname{csp} B$ from strain SM101 are indicated. (b) GUS specific activity from the $\operatorname{csp} B$-gus $A$ fusion in $C$. perfringens wild-type SM101 grown in TGY vegetative $(\triangle)$ and DS sporulation $(\boldsymbol{A})$ media was determined as described in Methods. Data are the mean of three independent experiments, and time zero denotes the time of inoculation of cells into either TGY or DS medium.

Construction of $\operatorname{csp} B-g u s A$ fusion plasmid and $\boldsymbol{\beta}$-glucuronidase (GUS) assay. A 441 bp DNA fragment upstream of $c s p B$ in $C$. perfringens SM101, which included the $170 \mathrm{bp}$ intergenic region between $\operatorname{csp} B$ and CPR2568 that most likely contains the $\operatorname{csp} B$ gene promoter, was PCR-amplified using primers CPP393/CPP397 (all primers used in this work are listed in Supplementary Table S2). The forward and reverse primers had SalI and PstI sites, respectively. This PCR fragment was digested with SalI and PstI and cloned between SalI and PstI sites in pMRS127 to create a $\operatorname{cspB}$ gusA fusion, giving plasmid pDP85. This plasmid was introduced by electroporation (Czeczulin et al., 1996) into C. perfringens SM101, and erythromycin-resistant $\left(\mathrm{Em}^{\mathrm{r}} ; 50 \mu \mathrm{g} \mathrm{ml}^{-1}\right)$ transformants were selected. Transformants carrying the $\operatorname{csp} B-g u s A$ fusion plasmid pDP85 were grown in TGY vegetative medium ( $3 \%$ trypticase soy, $2 \%$ glucose, $1 \%$ yeast extract, $0.1 \%$ L-cysteine) (Kokai-Kun et al., 1994) and in Duncan-Strong (DS) (Duncan \& Strong, 1968) sporulation medium, and assayed for GUS activity as described by Zhao \& Melville (1998). GUS specific activity was expressed in Miller units, which were calculated as described previously (Raju et al., 2006). 
Decoating treatment of sporulating cultures and spores prior to GUS assays. Cell pellets from $1 \mathrm{ml}$ of $8 \mathrm{~h}$ DS sporulating cultures or purified spore suspensions at $\mathrm{OD}_{600} 1.0$ were treated with $1 \mathrm{ml}$ $50 \mathrm{mM}$ Tris/HCl (pH 8.0), $8 \mathrm{M}$ urea, $1 \%$ (w/v) SDS and $50 \mathrm{mM}$ DTT for $90 \mathrm{~min}$ at $37{ }^{\circ} \mathrm{C}$, and remaining spores were washed three times with $150 \mathrm{mM} \mathrm{NaCl}$ and twice with water (Popham et al., 1995). The chemically decoated samples were then extracted and assayed for GUS activity as described above. Note that this urea-SDS treatment will inactivate or remove any GUS not in dormant spores, and will sensitize dormant spores to lysozyme, allowing the assay of enzymes in the dormant spore core (Mason et al., 1988; Paredes-Sabja et al., 2009b). GUS activities in these experiments were expressed relative to the amount in an equivalent volume of an untreated DS $8 \mathrm{~h}$ sporulating culture of the strain carrying the $\operatorname{csp} B-g u s A$ fusion, which was set to $100 \%$.

Construction of $\boldsymbol{C}$. perfringens $\operatorname{csp} B$ deletion mutant. To isolate a derivative of $C$. perfringens SM101 with a deletion of $\operatorname{csp} B$, a $\Delta \operatorname{csp} B$ suicide vector was constructed as follows. A 2016 bp DNA fragment carrying $112 \mathrm{bp}$ from the N-terminal coding region and $1949 \mathrm{bp}$ upstream of $\operatorname{csp} B$ was PCR-amplified using primers CPP356/CPP360 (forward and reverse primers had KpnI and SpeI cleavage sites, respectively, at the $5^{\prime}$ ends). A $1689 \mathrm{bp}$ fragment carrying $54 \mathrm{bp}$ from the C-terminal coding region and 1635 bp downstream of $\operatorname{csp} B$ was PCR-amplified using primers CPP359/CPP364 (forward and reverse primers had PstI and XhoI cleavage sites, respectively, at the $5^{\prime}$ ends). These PCR fragments were cloned into plasmid pCR-XL-TOPO (Invitrogen), giving plasmids pDP55 and pDP57, respectively. An $\sim 2.0 \mathrm{~kb}$ KpnI-SpeI fragment from pDP55 was cloned into pDP25 giving plasmid $\mathrm{pDP} 56$, and an $\sim 1.7 \mathrm{~kb}$ Pst $\mathrm{I}-\mathrm{Xho \textrm {I }}$ fragment from pDP57 was cloned into pDP56, giving pDP58. Next, an $\sim 5.0 \mathrm{~kb}$ $K p n \mathrm{I}-X h o \mathrm{I}$ fragment from pDP58 was cloned between the KpnI and SalI sites of pMRS104, giving pDP59. Finally, a $3.2 \mathrm{~kb} E c o$ RI fragment carrying tet $M$ was excised from pDP35, and the ends were filled and cloned into the HpaI site in catP of pDP59, giving plasmid pDP165, which cannot replicate in $C$. perfringens. Plasmid pDP165 was introduced into $C$. perfringens strain SM101 by electroporation (Czeczulin et al., 1996), and the $\operatorname{cspB}$ deletion strain DPS117 was isolated by allelic exchange (Sarker et al., 1999). The presence of the $\operatorname{csp} B$ deletion in strain DPS117 was confirmed by PCR and Southern blot analyses (results not shown).

Construction of a $\operatorname{cspB}$ mutant strain complemented with $\operatorname{cspB}$. To construct a $\operatorname{csp} B$ strain complemented with wild-type $\operatorname{csp} B$, a suicide-complementing plasmid targeted to the plc locus was constructed as follows. An $\sim 2.4 \mathrm{~kb}$ DNA fragment carrying $441 \mathrm{bp}$ from upstream and the coding region of $\operatorname{csp} B$ was PCR-amplified with Phusion High-Fidelity DNA polymerase (New England Biolabs) using primers CPP484/CPP485 (forward and reverse primers had KpnI and SalI sites, respectively, at their $5^{\prime}$ ends). As shown by assays of GUS activity (Fig. 1b), the 441 bp region upstream of $\operatorname{csp} B$ contains a sporulation-specific promoter. This PCR fragment was cloned into ZeroBlunt TOPO (Invitrogen), giving plasmid pDP121. Next, a $2.4 \mathrm{~kb} K p n \mathrm{I}-S a l \mathrm{I}$ fragment from pDP121 was cloned into the KpnI/ Sall sites of pDP129 [a suicide plasmid containing $\sim 1.7$ and $1.3 \mathrm{~kb}$ upstream and downstream, respectively, of the plc locus (ParedesSabja et al., 2009a)], giving plasmid pDP184, which cannot replicate in C. perfringens. Plasmid pDP184 was introduced into the $C$. perfringens $\operatorname{csp} B$ strain DPS117 by electroporation (Czeczulin et al., 1996) and $\mathrm{Em}^{\mathrm{r}} \mathrm{Tet}^{\mathrm{r}}$ transformants of strain DPS117(pDP184) were selected. The presence of both plasmid pDP184 and the original $\operatorname{csp} B$ deletion in the latter strain was confirmed by PCR and Southern blot analyses (data not shown).

Spore preparation and germination. Spores of various $C$. perfringens strains were prepared as previously described (ParedesSabja et al., 2008a, b, 2009a) and stored at $-20{ }^{\circ} \mathrm{C}$ until use. All spore preparations used in this work were $>99 \%$ pure as determined by phase-contrast microscopy. Spore germination was as described previously (Paredes-Sabja et al., 2008a, b, 2009a). Briefly, spore suspensions of $\mathrm{OD}_{600} 6$ were heat-activated at $80{ }^{\circ} \mathrm{C}$ for $10 \mathrm{~min}$, cooled in water at ambient temperature for $5 \mathrm{~min}$, and incubated at $40{ }^{\circ} \mathrm{C}$ for $10 \mathrm{~min}$. Germination of spores at $40{ }^{\circ} \mathrm{C}$ and $\mathrm{OD}_{600} 1$ with brain heart infusion (BHI) broth, $\mathrm{KCl}(100 \mathrm{mM} \mathrm{KCl}, 25 \mathrm{mM}$ sodium phosphate buffer, $\mathrm{pH} 7.0$ ), a mixture of $100 \mathrm{mM}$ L-asparagine, $100 \mathrm{mM} \mathrm{KCl}$ and $25 \mathrm{mM}$ sodium phosphate ( $\mathrm{pH} \mathrm{7.0)}$ (AK) or CaDPA ( $50 \mathrm{mM} \mathrm{CaCl}_{2}, 50 \mathrm{mM}$ DPA, adjusted to $\mathrm{pH} 8.0$ with Tris base) was routinely measured by monitoring the $\mathrm{OD}_{600}$ of spore cultures (Smartspec 3000 spectrophotometer, Bio-Rad Laboratories), which falls by $\sim 60 \%$ upon complete germination of wild-type spores. Levels of germination were also confirmed by phase-contrast microscopy. All values reported are averages of two experiments performed on at least two independent spore preparations, and individual values varied by less than $10 \%$ from average values shown.

Spore decoating treatment and assessment of colony forming ability. Spores at $\mathrm{OD}_{600} 20$ were decoated in $1 \mathrm{ml} 0.1 \mathrm{M}$ sodium borate (pH 10), $2 \%$ 2-mercaptoethanol for $60 \mathrm{~min}$ at $37^{\circ} \mathrm{C}$ (Miyata et al., 1997; Paredes-Sabja et al., 2008a), and the decoated spores were washed 10 times with distilled water before use.

To assess the colony forming efficiency of the spores, untreated and decoated spores at $\mathrm{OD}_{600} 1$ were heat-activated $\left(80{ }^{\circ} \mathrm{C}, 10 \mathrm{~min}\right)$, aliquots of various dilutions were plated on $\mathrm{BHI}$ agar with or without lysozyme $\left(1 \mu \mathrm{g} \mathrm{ml}^{-1}\right)$, the plates were incubated at $37{ }^{\circ} \mathrm{C}$ anaerobically for $24 \mathrm{~h}$, and colonies were counted.

Preparation of spore extracts and Western blot analysis. For preparation of coat extracts from dormant spores, aliquots $(200 \mu \mathrm{l})$ of spores $\left(\mathrm{OD}_{600}\right.$ 50) were decoated in $200 \mu \mathrm{l} 50 \mathrm{mM}$ Tris $/ \mathrm{HCl}$ $(\mathrm{pH} 8.0), 8 \mathrm{M}$ urea, $1 \%(\mathrm{w} / \mathrm{v})$ SDS and $50 \mathrm{mM}$ DTT for $90 \mathrm{~min}$ at $37^{\circ} \mathrm{C}$, centrifuged (13200 r.p.m.) for $5 \mathrm{~min}$, and the supernatant fluid containing coat material from dormant spores was stored at $-20{ }^{\circ} \mathrm{C}$ until use. For preparation of germinated spores, aliquots $\left(200 \mu \mathrm{l}\right.$ at $\left.\mathrm{OD}_{600} 250\right)$ of heat-activated spores were suspended at $\mathrm{OD}_{600} 250$ in $200 \mu \mathrm{l} 25 \mathrm{mM}$ sodium phosphate ( $\mathrm{pH} \mathrm{7.0),} 100 \mathrm{mM}$ $\mathrm{KCl}$, germinated for $2 \mathrm{~h}$ at $37{ }^{\circ} \mathrm{C}$, and stored at $-20{ }^{\circ} \mathrm{C}$ until use. For preparation of coat extracts from germinated $\operatorname{csp} B$ spores, aliquots $(200 \mu \mathrm{l})$ of germinated spores $\left(\mathrm{OD}_{600} 250\right)$ were decoated as described above, and the supernatant fluid was stored at $-20{ }^{\circ} \mathrm{C}$ until use.

Samples $(10 \mu \mathrm{l})$ of coat extracts or intact germinated spores were boiled in SDS-PAGE loading buffer and run on SDS-PAGE gels (12\% acrylamide), and proteins were transferred to a PVDF membrane (Millipore). These Western blots were probed with a $1: 10000$ dilution of anti-SleC polyclonal antibody (Miyata et al., 1995) for $1 \mathrm{~h}$ at room temperature and then with a 1:10000 dilution of goat antimouse IgG-horseradish peroxidase (HRP) conjugate (Promega) for $1 \mathrm{~h}$ at room temperature, in PBS ( $25 \mathrm{mM}$ sodium phosphate, $\mathrm{pH} 7.4$, $150 \mathrm{mM} \mathrm{NaCl}$ ) with $1 \%$ BSA and $0.05 \%$ Tween. HRP activity was detected with a chemiluminescence detection system (Molecular Imager ChemiDoc XRS + System, Bio-Rad) by using the PicoMax sensitive chemiluminescent HRP substrate (Rockland Immunochemicals). Each Western blot also included $5 \mu$ l PageRuler Plus prestained Protein Ladder (Fermentas).

Analytical procedures. DPA remaining in spores during and after germination was measured as described previously (Paredes-Sabja et al., 2008a, b, 2009a). Briefly, heat-activated spore suspensions $\left(\mathrm{OD}_{600}\right.$ 1.5) were cooled and incubated at $40{ }^{\circ} \mathrm{C}$ in $25 \mathrm{mM}$ sodium phosphate buffer $(\mathrm{pH} \mathrm{7.0)}$ or in $\mathrm{KCl}$, as described above. After 1 or $24 \mathrm{~h}$, aliquots $(1 \mathrm{ml})$ were centrifuged in a microcentrifuge (13200 r.p.m., $3 \mathrm{~min}$ ), and the spore pellet was washed twice with $1 \mathrm{ml}$ distilled water and suspended in $1 \mathrm{ml}$ distilled water. The remaining spore 
DPA was determined by boiling samples for $60 \mathrm{~min}$, cooling on ice for $5 \mathrm{~min}$, centrifuging in a microcentrifuge for $5 \mathrm{~min}$, and measuring the $\mathrm{OD}_{270}$ of the supernatant fluid as described previously (ParedesSabja et al., 2009a). The initial DPA content of dormant spores was determined by boiling $1 \mathrm{ml}$ aliquots for $60 \mathrm{~min}$, centrifuging in a microcentrifuge (13200 r.p.m., $5 \mathrm{~min}$ ), and measuring the $\mathrm{OD}_{270}$ of the supernatant fluid as described previously (Paredes-Sabja et al., $2008 \mathrm{a}, \mathrm{b}, 2009 \mathrm{a})$. In C. perfringens spores, $\sim 90 \%$ of the material absorbing at $270 \mathrm{~nm}$ released from spores by boiling is DPA (ParedesSabja et al., 2009a).

The release of fragments of cortex PG containing hexosamine during germination was measured by germinating heat-activated spores at $\mathrm{OD}_{600} 25$ in $100 \mathrm{mM} \mathrm{KCl}$ and $10 \mathrm{mM}$ Tris/ $\mathrm{HCl}$ (pH 7.4). After $2 \mathrm{~h}$ incubation at $40{ }^{\circ} \mathrm{C}$, samples $(1 \mathrm{ml})$ were centrifuged (13 200 r.p.m., $5 \mathrm{~min}$ ), and analyses of hexosamine in the supernatant fluid were carried out as described previously (Ghuysen et al., 1966; ParedesSabja et al., 2009a). Analysis of total hexosamine-containing material in dormant spores was also carried out as described previously (Ghuysen et al., 1966; Paredes-Sabja et al., 2009a).

\section{RESULTS}

\section{Arrangement of the csp-s/eC locus and expression of $\operatorname{cspB}$ in C. perfringens SM101}

Earlier studies (Masayama et al., 2006; Shimamoto et al., 2001) with C. perfringens S40 indicate that three Csp proteases (CspA, B and C) belonging to the subtilisin family of serine proteases are encoded by a tricistronic operon upstream of the sleC gene (Paredes-Sabja et al., 2009a). However, in the FP isolate SM101, there is only one $\operatorname{csp}, \operatorname{csp} B$, upstream of $\operatorname{sle} C$ (Fig. 1a). Amino acid sequence alignments indicate that $\mathrm{CspB}^{\mathrm{SM101}}$ shares high homology [92\% identity (I) and $96 \%$ similarity (S)] to $\mathrm{CspB}^{\mathrm{S40}}$, with much lower homology to $\mathrm{CspA}^{\mathrm{S} 40}(32 \% \mathrm{I}, 50 \% \mathrm{~S})$ and $\mathrm{CspC}^{\mathrm{S} 40}$ (31\% I, $51 \%$ S) (Supplementary Fig. S1).

To determine whether $\operatorname{cs} p B$ is expressed during sporulation in $C$. perfringens SM101, upstream DNA from $\operatorname{csp} B$, including the intergenic region between $\operatorname{csp} B$ and the preceding gene (Fig. 1a), which most likely contains the $\operatorname{csp} B$ gene promoter, was fused to E. coli gusA, and GUS activity was measured after introducing the fusion into $C$. perfringens SM101. Vegetative cultures of SM101 carrying the $\operatorname{csp} B-g u s A$ fusion had no significant GUS activity (Fig. $1 b$ ), and there was also no detectable GUS activity in sporulating cultures of strain SM101 without the $\operatorname{csp} B$ gusA fusion (data not shown). However, there was significant GUS activity in sporulating cultures carrying the $\operatorname{csp} B-g u s A$ fusion (Fig. 1b), indicating that a sporulation-specific promoter is located upstream of $\operatorname{csp} B$. GUS expression from this putative $\operatorname{csp} B$ promoter appeared $\sim 4 \mathrm{~h}$ after the start of sporulation, and reached a maximum after $10 \mathrm{~h}$ (Fig. 1b). To evaluate the site of expression of $\operatorname{csp} B$, sporulating cultures and purified spores of strain SM101(pDP85) (wild-type carrying the cspB-gusA fusion) were treated with a decoating regime (Popham et al., 1995) that inactivates mother cell enzyme activity but has no effect on enzymes within the spore core. Although significant GUS activity was detected in untreated spor- ulating cultures of the strain carrying $\operatorname{csp} B-g u s A$ as expected, no significant GUS activity was detected in decoated sporulating cultures and either untreated or decoated purified spores (data not shown). These results clearly indicate that $\operatorname{csp} B$ is expressed uniquely in the mother cell compartment of the sporulating cell.

\section{Effect of $\operatorname{cspB}$ deletion on SM101 spore germination and colony formation with BHI broth}

Since $\operatorname{csp} B$ is expressed during sporulation, we hypothesized that $\operatorname{csp} B$ is essential for pro-SleC processing and therefore for cortex hydrolysis during germination of spores of $C$. perfringens SM101. Consequently, we constructed a $\operatorname{csp} B$ deletion (strain DPS117) (Fig. 2a). As expected, wild-type spores germinated well in BHI broth as measured by changes in the $\mathrm{OD}_{600}$ of the germinating culture (Fig. 2b). However, $\operatorname{csp} B$ spores exhibited little if any germination in BHI broth, and their germination was even less than that of wild-type spores in phosphate buffer as measured by the fall in $\mathrm{OD}_{600}$ (Fig. 2b). Phase-contrast microscopy also showed that $>90 \%$ of wild-type spores had become phase dark after $60 \mathrm{~min}$ in BHI broth, indicating that the great majority of these spores had completed germination, while $\operatorname{csp} B$ spores remained phase bright (data not shown), indicative of no germination. The germination defect of $\operatorname{csp} B$ spores was complemented by ectopic insertion of a wild-type $\operatorname{csp} B$ gene in the $\operatorname{csp} B$ strain, indicating that the germination defect in $\operatorname{csp} B$ spores is due to specific inactivation of $\operatorname{csp} B$ (Fig. 2b). These results suggest that $\operatorname{csp} B$ is essential for germination of spores of $C$. perfringens $\mathrm{FP}$ isolates.

The severity of the germination defect of $\operatorname{csp} B$ spores suggested that, as found for $s l e C$ spores (Paredes-Sabja et al., 2009a), the colony forming efficiency of $\operatorname{csp} B$ spores is lower than that of wild-type spores, since Csp proteins appear to be required for activation of the major $C$. perfringens CLE, SleC (Paredes-Sabja et al., 2009a; Shimamoto et al., 2001). Indeed, the colony forming efficiency of $\operatorname{csp} B$ spores was $\sim 10^{4}$-fold lower than that of wild-type spores (Table 1). However, this defect was eliminated when $\operatorname{csp} B$ spores were decoated and plated on BHI agar plates containing lysozyme (Table 1), indicating that $\operatorname{csp} B$ spores were viable, but probably incapable of degrading their PG cortex, as noted previously for sleC spores (Paredes-Sabja et al., 2009a). The latter result also suggests that active SleC is not generated during germination of $\operatorname{csp} B$ spores, since SleC alone is essential for cortex degradation during spore germination, and thus full spore viability (Paredes-Sabja et al., 2009a). The absence of active $\mathrm{SleC}$ in $\operatorname{csp} B$ spores could be due to lack of conversion of pro-SleC to SleC during germination (see below). However, since the low viability of $\operatorname{csp} B$ spores on plates without lysozyme was complemented by wild-type $\operatorname{csp} B$ inserted in the plc locus (Table 1), it appears most likely that pro-SleC is present in $\operatorname{csp} B$ spores but cannot be converted to active SleC during germination. Interestingly, incubation of $\operatorname{csp} B$ spores on BHI plates without lysozyme 
(a)

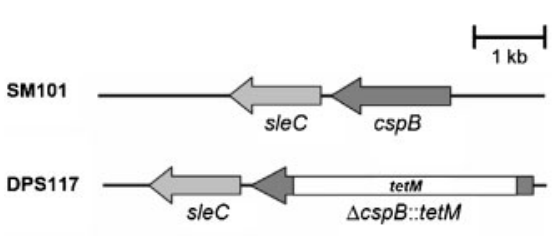

(b)

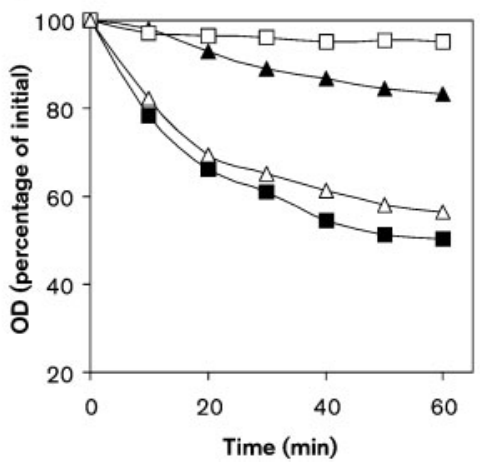

(c)

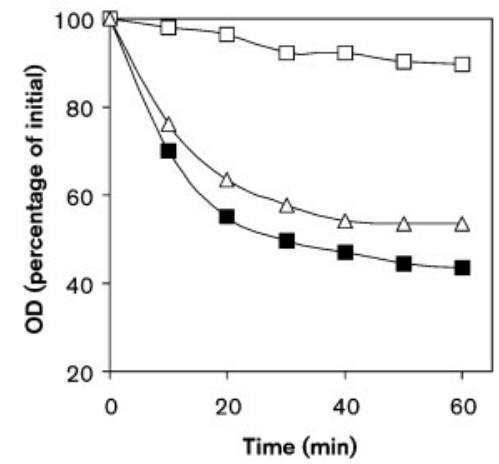

Fig. 2. (a) Schematic representation of $\operatorname{cspB}$ deletion mutant in C. perfringens SM101 and (b, c) germination of mutant spores in $\mathrm{BHI}$ broth. (a) Arrangement of $\operatorname{cspB}$ in the C. perfringens wild-type and $\operatorname{csp} B$ mutant strains. (b, c) Heat-activated spores of C. perfringens strains SM101 (wild-type) ( $\mathbf{\square})$, DPS117 (cspB) ( $\square$ ) and DPS117 (pDP184) (cspB mutant complemented with wild-type $\operatorname{csp} B)(\triangle)$ were germinated at $40{ }^{\circ} \mathrm{C}$ in (b) BHI broth or (c) Ca-DPA, and the $\mathrm{OD}_{600}$ was measured as described in Methods. Heat-activated spores of SM101 (wild-type) (A) were germinated at $40{ }^{\circ} \mathrm{C}$ in $25 \mathrm{mM}$ sodium phosphate (pH 7.0) (b).

for long periods of time did slowly give rise to colonies in a manner similar to that previously reported for sleC spores (Paredes-Sabja et al., 2009a), i.e. some colonies started to appear only surrounding those colonies that appeared within the first $24 \mathrm{~h}$, suggesting that a cell wall hydrolase released from growing or sporulating cells was diffusing through the medium and germinating a small percentage of $\operatorname{csp} B$ spores that have a defective coat.

In Bacillus subtilis and Bacillus megaterium, Ca-DPA triggers germination by activation of the CLE CwlJ (Paidhungat et al., 2001; Setlow et al., 2009). In contrast, recent work (Paredes-Sabja et al., 2008b, 2009a) suggests that Ca-DPA triggers $C$. perfringens spore germination

Table 1. Colony formation by spores of $C$. perfringens strains

Heat-activated spores of various strains were plated on BHI agar with or without lysozyme, and colonies were counted after incubation at $37{ }^{\circ} \mathrm{C}$ for $24 \mathrm{~h}$.

\begin{tabular}{|lcc|}
\hline Strain/genotype & \multicolumn{2}{c|}{$\begin{array}{c}\text { Spore titre (c.f.u. } \mathbf{~ m l}^{-1} \\
\text { per } \text { OD }_{\mathbf{6 0 0}} \text { unit }\end{array}$} \\
\cline { 2 - 3 } & BHI & BHI+ Lyz $\dagger$ \\
\hline SM101/wild-type & $4.1 \times 10^{7}$ & $6.6 \times 10^{7}$ \\
DPS117/cspB & $3.8 \times 10^{3}$ & $6.0 \times 10^{7}$ \\
DPS117(pDP184) cspB carrying & $5.3 \times 10^{7}$ & $\mathrm{ND} \ddagger$ \\
wild-type $\operatorname{csp} B$ & & \\
\hline
\end{tabular}

${ }^{*}$ Titres are the mean c.f.u. $\mathrm{ml}^{-1}$ per $\mathrm{OD}_{600}$ unit determined in three experiments and the variance was less than $25 \%$.

$\dagger$ Spores were decoated and plated on BHI plates containing $1 \mu \mathrm{g}$ lysozyme $\mathrm{ml}^{-1}$.

$\$ N D$, Not determined. through the GerKA-KC receptor and not by activating either pro-SleC or SleC. Since $\operatorname{csp} B$ spores possess pro-SleC (see below), we sought to provide definitive evidence for this suggestion by examining the germination of wild-type, cspB and DPS117(pDP184) (cspB mutant complemented with wild-type $\operatorname{csp} B$ ) spores with exogenous Ca-DPA. As expected, wild-type spores germinated well with exogenous Ca-DPA, while $\operatorname{csp} B$ spores exhibited little if any germination, as assessed either by the decrease in $\mathrm{OD}_{600}$ of germinating cultures or by phase-contrast microscopy (Fig. $2 \mathrm{c}$ and data not shown). However, the Ca-DPA germination defect in $\operatorname{csp} B$ spores was complemented by an ectopic copy of $\operatorname{csp} B$ (Fig. 2c and data not shown). These results suggest that Ca-DPA does not initiate germination by activating pro-SleC, but rather presumably through the GerKA-KC receptor, as suggested previously (ParedesSabja et al., 2008b).

\section{CspB is essential for processing of pro-SleC into mature SleC during spore germination}

To provide definitive evidence that the germination defect of $\operatorname{csp} B$ spores is due to lack of processing of inactive pro-SleC into active SleC, spore extracts were analysed by Western blotting with anti-SleC antibody (Fig. 3a). As expected (Miyata et al., 1997), coat extracts of dormant wild-type spores had pro-SleC but not SleC, while wildtype germinated spores had pro-SleC and a fraction processed into the smaller SleC (Fig. 3a). Coat extracts from dormant $\operatorname{csp} B$ spores also had pro-SleC, but there was no detectable processing of pro-SleC into SleC either in intact germinated $c s p B$ spores or in coat extracts from these spores (Fig. 3a). This defect was complemented by an ectopic copy of $\operatorname{csp} B$ (Fig. 3a). These results indicate that $\mathrm{CspB}$ is essential for processing of inactive pro-SleC into 
(a)

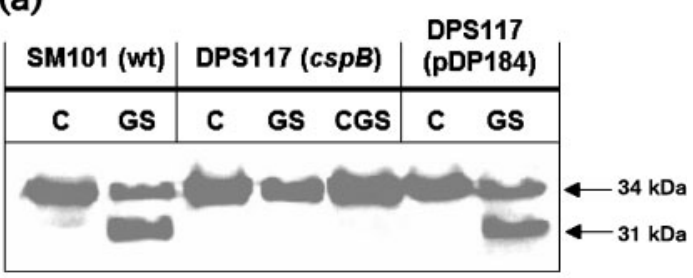

(b)

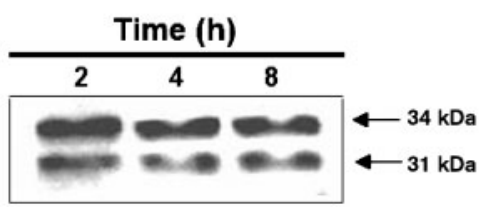

Fig. 3. Detection of SleC in C. perfringens spores. (a) Aliquots of coat extracts from dormant (C) and germinated spores (CGS), and intact germinated spores (GS) were separated by SDS-PAGE, proteins were transferred to a PVDF membrane, and SleC was detected with anti-SleC antibody as described in Methods. Samples run in various lanes were from spores of strains SM101 (wild-type), DPS117 (cspB mutant) and DPS117(pDP184) (cspB complemented with wild-type $\operatorname{csp} B$ ), and the arrows on the right indicate the predicted migration positions of pro-SleC (34 kDa) and SleC (31 kDa) (Miyata et al., 1995). (b) Spores of strain SM101 (wild-type) were germinated with $100 \mathrm{mM} \mathrm{KCl}$ for 2, 4 and $8 \mathrm{~h}$, the intact spore was extracted, aliquots were run on SDSPAGE, and SleC was detected by Western blot analysis as described for (a).

active SleC early during germination, and that pro-SleC is present in $\operatorname{csp} B$ spores.

It was somewhat surprising that only a fraction of pro-SleC was processed into SleC by CspB during $2 \mathrm{~h}$ of germination of C. perfringens SM101 as well as DPS117(pDP184) ( $\operatorname{csp} B$ complemented with an ectopic copy of $c s p B$ ) with $\mathrm{KCl}$, since $\sim 99 \%$ of spores of both strains became phase dark after $2 \mathrm{~h}$ of germination (data not shown). To evaluate whether further incubation was required to complete pro-SleC processing, wild-type spores were incubated for 2, 4 and $8 \mathrm{~h}$ with $\mathrm{KCl}$. However, the fraction of pro-SleC converted to active SleC increased only slightly upon extended incubation (Fig. 3b). Similar results were observed using the germinant AK (data not shown). These results suggest that only a fraction of inactive pro-SleC needs to be converted into active $\mathrm{SleC}$ by $\mathrm{CspB}$ to trigger full germination of spores of $C$. perfringens $\mathrm{FP}$ isolates.

\section{CspB is essential for initiation of cortex hydrolysis during germination of $C$. perfringens spores}

Previously we reported that during C. perfringens spore germination, sleC spores release their DPA slower than wild-type spores and are unable to degrade their PG cortex (Paredes-Sabja et al., 2009a). To gain more insight into the role of CspB in C. perfringens spore germination, wild-type,
$\operatorname{cspB}$ and DPS117(pDP184) spores were germinated with $\mathrm{KCl}$ (Paredes-Sabja et al., 2008b). Wild-type spores germinated completely with $\mathrm{KCl}$, while $\operatorname{csp} B$ spores showed a minimal fall in $\mathrm{OD}_{600}$ upon incubation with $\mathrm{KCl}$, although this defect was eliminated in the $\operatorname{csp} B$ strain complemented with an ectopic copy of $\operatorname{csp} B$ (Fig. 4a). Phase-contrast microscopy further showed that $>99 \%$ of wild-type and DPS117(pDP184) spores had become phase dark after $1 \mathrm{~h}$ of $\mathrm{KCl}$ germination, while the $\operatorname{csp} B$ spores remained phase bright (data not shown). The phasecontrast microscopy results and the lack of fall in the $\mathrm{OD}_{600}$ of $c s p B$ spores suggested that little if any DPA was released during $\mathrm{KCl}$ germination of these spores. To directly confirm this conclusion, we measured DPA remaining in $\mathrm{KCl}$-germinated wild-type, $\operatorname{csp} B$ and DPS117(pDP184) spores. After $1 \mathrm{~h}$ of incubation in $\mathrm{KCl}$, wild-type spores had released most of their DPA (Fig. 4b). However, the amount of DPA released from $\operatorname{csp} B$ spores after $1 \mathrm{~h}$ of incubation with $\mathrm{KCl}$ was minimal (Fig. 4b), and similar to that released from wild-type spores incubated for $1 \mathrm{~h}$ at $40{ }^{\circ} \mathrm{C}$ in sodium phosphate buffer alone (data not shown), in which $C$. perfringens spores germinate minimally if at all. Incubation of $\operatorname{csp} B$ spores in $\mathrm{KCl}$ for $24 \mathrm{~h}$ led to only an insignificant increase in the amount of DPA released (Fig. 4b). However, DPA release during $\mathrm{KCl}$ germination was restored in $\operatorname{csp} B$ spores upon their complementation with wild-type $\operatorname{csp} B$ (Fig. 4b).

The culminating event in spore germination is the hydrolysis of the spore PG cortex and release of PG fragments into the medium. Therefore, we monitored cortex hydrolysis by assaying the release of hexosaminecontaining material into the medium (Ghuysen et al., 1966; Paredes-Sabja et al., 2009a). As expected, wild-type spores released significant amounts of hexosamine-containing material into the medium after $2 \mathrm{~h}$ of germination with $\mathrm{KCl}$ (Fig. 4c). In contrast, hexosamine-containing material was not released by $\operatorname{csp} B$ spores during $\mathrm{KCl}$ germination, although this release was restored in $\operatorname{csp} B$ spores complemented with wild-type $\operatorname{csp} B$ (Fig. 4c). These results clearly indicate that CspB is essential for cortex hydrolysis during $C$. perfringens spore germination through the processing of pro-SleC into mature SleC.

\section{DISCUSSION}

Results presented in this paper provide strong support for the hypothesis that Csp proteases are essential in germination of $C$. perfringens spores in order to activate pro-SleC and thus allow cortex PG degradation. This has been suggested previously based on results from in vitro studies of the effects of Csp proteins on pro-SleC and the activity of pro-SleC and SleC on cortex PG in decoated spores (Okamura et al., 2000; Urakami et al., 1999). The current work shows that $C$. perfringens spores lacking their sole Csp protein, CspB, cannot germinate with all germinants tested. In addition, these $\operatorname{csp} B$ spores had extremely low viability, most likely because of their poor 
(a)

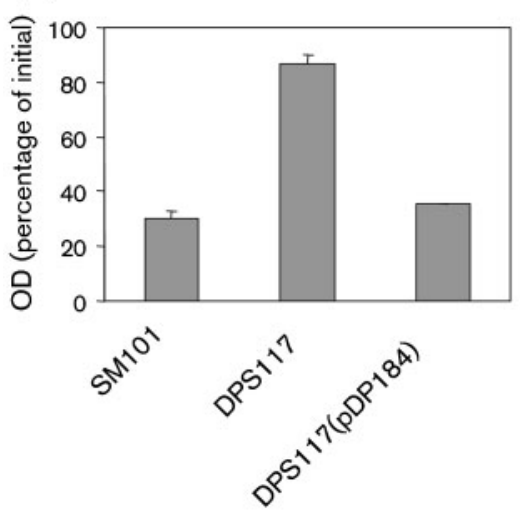

(b)

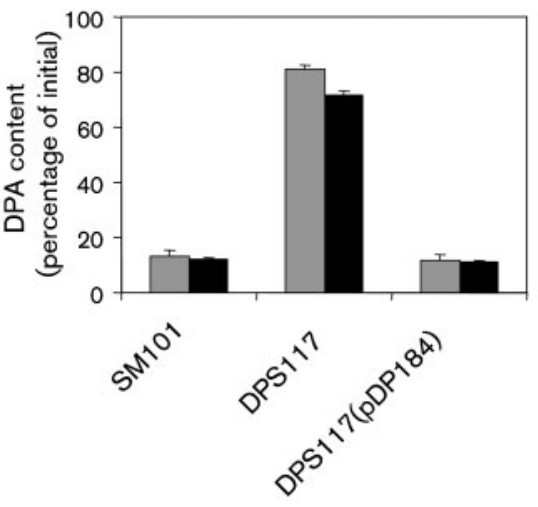

(c)

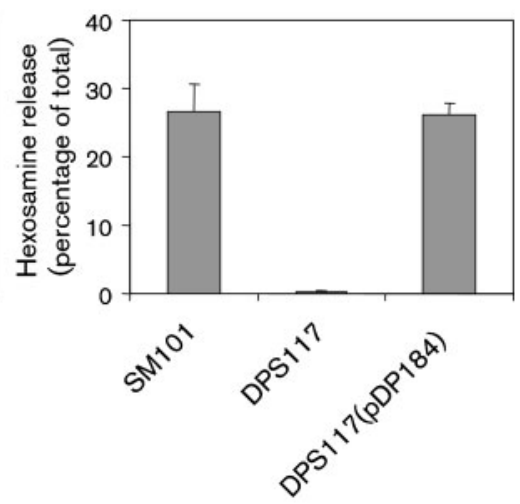

Fig. 4. Germination of spores of $C$. perfringens strains with $\mathrm{KCl}$. (a) Heat-activated C. perfringens spores of strains $\mathrm{SM} 101$ (wild-type), DPS117 ( $c s p B$ ) and DPS117(pDP184) (cspB mutant complemented with $c s p B$ ) were germinated with $\mathrm{KCl}$, and the $\mathrm{OD}_{600}$ was measured after $1 \mathrm{~h}$ as described in Methods. (b) DPA release during $C$. perfringens spore germination with $\mathrm{KCl}$. Heat-activated spores of $C$. perfringens strains were germinated with $\mathrm{KCl}$ and after $1 \mathrm{~h}$ (grey bars) and $18 \mathrm{~h}$ (black bars) the DPA content of spores was measured as described in Methods. DPA release from spores of $C$. perfringens strains incubated at $40{ }^{\circ} \mathrm{C}$ in $25 \mathrm{mM}$ sodium phosphate buffer (pH 7.0) was less than $20 \%$. (c) Release of hexosamine-containing material during C. perfringens spore germination with $\mathrm{KCl}$. Heat-activated spores of $\mathrm{C}$. perfringens strains were germinated with $\mathrm{KCl}$ for $2 \mathrm{~h}$ and the hexosamine-containing material released into the medium was measured as described in Methods. Values of hexosamine-containing material released are expressed relative to the amount of hexosamine in dormant spores, which was defined as $100 \%$. Data represent the mean of three independent experiments with two different spore preparations; error bars, SD.

germination. The fact that the low viability of $\operatorname{csp} B$ spores could be corrected if these spores were decoated and applied to plates containing lysozyme is consistent with the defect in the germination of $\operatorname{csp} B$ spores being in cortex degradation, and thus that the function of CspB is to convert inactive pro-SleC into active SleC that can degrade cortex PG. When this work was begun, it was considered possible that a $\operatorname{csp} B$ deletion somehow prevents sle $C$ expression, and that $\operatorname{csp} B$ spores lack even pro-SleC; since $\operatorname{csp} B$ is just upstream of $s l e C$, a $\operatorname{csp} B$ deletion might interfere with sle $C$ transcription somehow, perhaps even transcription from a promoter upstream of $\operatorname{csp} B$. However, this possibility seemed unlikely for a variety of reasons including: (i) an ectopic sleC with only upstream DNA from the intergenic region between $\operatorname{csp} B$ and $\operatorname{sle} C$ can completely complement the spore germination defects of a sleC deletion strain (Paredes-Sabja et al., 2009a); (ii) there is a very stable stem-loop structure encoded in the intergenic region between $\operatorname{csp} B$ and $s l e C$, and it appears likely that this is a strong terminator of $\operatorname{csp} B$ transcription with a predicted $\Delta G$ of $-6.3 \mathrm{kcal} \mathrm{mol}^{-1}\left(-26.4 \mathrm{~kJ} \mathrm{~mol}^{-1}\right)$ [determined using mfold (http://mfold.bioinfo.rpi.edu/cgibin/rna-form1.cgi)]; and (iii) there is a strong promoter in the intergenic region between $\operatorname{csp} B$ and $s l e C$ that drives sporulation-specific sleC expression (Paredes-Sabja et al., 2009a). In addition, as shown in the current work: (iv) ectopic expression of $\operatorname{csp} B$ completely complemented the germination and viability defects in $\operatorname{csp} B$ spores; and (v) the presence of pro-SleC in $\operatorname{csp} B$ spores, and at levels similar to those in wild-type spores, was shown in this work by Western blot analysis.

Since $\operatorname{csp} B$ spores have normal levels of pro-SleC, as noted above, it seems likely that it is CspB that processes this zymogen and thus triggers cortex hydrolysis during spore germination. Interestingly, only a fraction of total pro-SleC is processed to active $\mathrm{SleC}$ by $\mathrm{CspB}$ during spore germination, but this fractional amount of active SleC is sufficient for complete germination of $C$. perfringens spores. The obvious question is then what triggers $\mathrm{CspB}$ activity during spore germination, as Csp proteins appear to be present in spores in a potentially active form. Unfortunately, there is no information available on the regulation of the activity of CspB, or Csp proteases in general, and this is clearly an important matter for further study.

While it appears clear that CspB is essential for spore germination by activating pro-SleC, it is possible that $\mathrm{CspB}$ has some other function and/or substrate. The evidence for this is that the sole function of CspB is to activate pro-SleC, then the germination phenotype of $\operatorname{csp} B$ spores should be identical to that of $s l e C$ spores. Indeed, the viability of sleC spores is $10^{4}$-fold lower than that of wild-type spores and sleC spores germinate very poorly with all germinants tested (Paredes-Sabja et al., 2009a), as is also the case for $\operatorname{csp} B$ spores. However, while sle $C$ spores cannot complete germination very efficiently because they cannot degrade their PG cortex, they slowly release much of their Ca-DPA 
during germination with a number of germinants (Paredes-Sabja et al., 2009a). In contrast, cspB spores do not. Thus, $\operatorname{csp} B$ spores have a more severe germination phenotype than sleC spores, consistent with CspB having some germination-associated function in addition to processing pro-SleC. However, the nature of this additional function as well as that of other possible CspB substrates in spores is not known, and these topics are also matters for further work.

The involvement of CspB in pro-SleC activation and thus cortex hydrolysis is a further example of the differences in the signalling pathways in the germination of spores of Clostridium and Bacillus species. In Bacillus species, spores have two redundant CLEs, CwlJ and SleB, and both are present in spores in a mature form that is activated during germination either by Ca-DPA released from the spore core (CwlJ), or perhaps by some drastic change in strain on the cortical PG following Ca-DPA release and its replacement by water in the spore core (SleB) (Paidhungat et al., 2001; Setlow et al., 2009; Setlow, 2003). In further contrast to the situation in Clostridium spores, there is no known involvement of Csp proteases, or indeed of any protease at all in the regulation of CLE activity in spores of Bacillus species.

The final conclusion from the current work concerns the difference in the number of Csp proteases in the FP and non-FP strains, one in the former and three in the latter. While it is formally possible that three Csp proteases are needed for full SleC processing, including removal of the $\mathrm{N}$ and C-terminal pre-sequences (Urakami et al., 1999), clearly a single Csp is sufficient to generate enough active SleC in strain SM101 to allow complete germination. Thus there appears to be no clear necessity for three Csp proteases for the germination of $C$. perfringens spores. However, it is certainly possible that the additional two Csp proteases in non-FP isolates have some additional roles in spore germination, and this too is a matter for further study.

\section{ACKNOWLEDGEMENTS}

This work was supported by a grant from the N. L. Tartar Foundation of Oregon State University, by a grant from the Agricultural Research Foundation of Oregon State University (to M.R.S.), and by a grant from the Army Research Office to M. R.S. and P.S. We are grateful to Ryuichi Moriyama (Department of Food and Nutritional Sciences, Chubu University, Japan) for the gift of the antibody against SleC.

\section{REFERENCES}

Cowan, A. E., Koppel, D. E., Setlow, B. \& Setlow, P. (2003). A soluble protein is immobile in dormant spores of Bacillus subtilis but is mobile in germinated spores: implications for spore dormancy. Proc Natl Acad Sci U S A 100, 4209-4214.

Czeczulin, J. R., Collie, R. E. \& McClane, B. A. (1996). Regulated expression of Clostridium perfringens enterotoxin in naturally cpenegative type A, B, and C isolates of C. perfringens. Infect Immun 64, 3301-3309.
Duncan, C. L. \& Strong, D. H. (1968). Improved medium for sporulation of Clostridium perfringens. Appl Microbiol 16, 82-89.

Ghuysen, J. M., Tipper, D. J. \& Strominger, J. L. (1966). Enzymes that degrade bacterial cell walls. Methods Enzymol 8, 685-699.

Kokai-Kun, J. F., Songer, J. G., Czeczulin, J. R., Chen, F. \& McClane, B. A. (1994). Comparison of Western immunoblots and gene detection assays for identification of potentially enterotoxigenic isolates of Clostridium perfringens. J Clin Microbiol 32, 2533-2539.

Kumazawa, T., Masayama, A., Fukuoka, S., Makino, S., Yoshimura, T. \& Moriyama, R. (2007). Mode of action of a germination-specific cortex-lytic enzyme, SleC, of Clostridium perfringens S40. Biosci Biotechnol Biochem 71, 884-892.

Masayama, A., Hamasaki, K., Urakami, K., Shimamoto, S., Kato, S., Makino, S., Yoshimura, T., Moriyama, M. \& Moriyama, R. (2006). Expression of germination-related enzymes, CspA, CspB, CspC, SleC, and SleM, of Clostridium perfringens S40 in the mother cell compartment of sporulating cells. Genes Genet Syst 81, 227-234.

Mason, J. M., Hackett, R. H. \& Setlow, P. (1988). Regulation of expression of genes coding for small, acid-soluble proteins of Bacillus subtilis spores: studies using lacZ gene fusions. J Bacteriol 170, 239244.

McClane, B. A. (2007). Clostridium perfringens. In Food Microbiology: Fundamentals and Frontiers, pp. 423-444. Edited by M. P. Doyle \& L. R. Beuchat. Washington, DC: American Society for Microbiology. McDonnell, J. L. (1986). Toxins of Clostridium perfringens type A, B, C, D, and E. In Pharmacology of Bacterial Toxins, pp. 477-517. Edited by F. Dorner \& J. Drews. Oxford: Pergamon Press.

Miyata, S., Moriyama, R., Miyahara, N. \& Makino, S. (1995). A gene (sleC) encoding a spore-cortex-lytic enzyme from Clostridium perfringens S40 spores; cloning, sequence analysis and molecular characterization. Microbiology 141, 2643-2650.

Miyata, S., Kozuka, S., Yasuda, Y., Chen, Y., Moriyama, R., Tochikubo, K. \& Makino, S. (1997). Localization of germinationspecific spore-lytic enzymes in Clostridium perfringens S40 spores detected by immunoelectron microscopy. FEMS Microbiol Lett 152, 243-247.

Myers, G. S., Rasko, D. A., Cheung, J. K., Ravel, J., Seshadri, R., DeBoy, R. T., Ren, Q., Varga, J., Awad, M. M. \& other authors (2006). Skewed genomic variability in strains of the toxigenic bacterial pathogen, Clostridium perfringens. Genome Res 16, 1031-1040.

Okamura, S., Urakami, K., Kimata, M., Aoshima, T., Shimamoto, S., Moriyama, R. \& Makino, S. (2000). The N-terminal prepeptide is required for the production of spore cortex-lytic enzyme from its inactive precursor during germination of Clostridium perfringens S40 spores. Mol Microbiol 37, 821-827.

Paidhungat, M., Ragkousi, K. \& Setlow, P. (2001). Genetic requirements for induction of germination of spores of Bacillus subtilis by $\mathrm{Ca}^{2+}$-dipicolinate. J Bacteriol 183, 4886-4893.

Paredes-Sabja, D., Setlow, B., Setlow, P. \& Sarker, M. R. (2008a). Characterization of Clostridium perfringens spores that lack SpoVA proteins and dipicolinic acid. J Bacteriol 190, 4648-4659.

Paredes-Sabja, D., Torres, J. A., Setlow, P. \& Sarker, M. R. (2008b). Clostridium perfringens spore germination: characterization of germinants and their receptors. J Bacteriol 190, 1190-1201.

Paredes-Sabja, D., Setlow, P. \& Sarker, M. R. (2009a). SleC is essential for cortex peptidoglycan hydrolysis during germination of spores of the pathogenic bacterium Clostridium perfringens. J Bacteriol 191, 2711-2720.

Paredes-Sabja, D., Setlow, P. \& Sarker, M. R. (2009b). GerO, a putative $\mathrm{Na}^{+} / \mathrm{H}^{+}-\mathrm{K}^{+}$antiporter, is essential for normal germination of spores of the pathogenic bacterium Clostridium perfringens. J Bacteriol 191, 3822-3831. 
Popham, D. L., Sengupta, S. \& Setlow, P. (1995). Heat, hydrogen peroxide, and UV resistance of Bacillus subtilis spores with increased core water content and with or without major DNA-binding proteins. Appl Environ Microbiol 61, 3633-3638.

Popham, D. L., Helin, J., Costello, C. E. \& Setlow, P. (1996). Muramic lactam in peptidoglycan of Bacillus subtilis spores is required for spore outgrowth but not for spore dehydration or heat resistance. Proc Natl Acad Sci U S A 93, 15405-15410.

Raju, D., Waters, M., Setlow, P. \& Sarker, M. R. (2006). Investigating the role of small, acid-soluble spore proteins (SASPs) in the resistance of Clostridium perfringens spores to heat. BMC Microbiol 6, 50.

Sarker, M. R., Carman, R. J. \& McClane, B. A. (1999). Inactivation of the gene $(c p e)$ encoding Clostridium perfringens enterotoxin eliminates the ability of two cpe-positive $C$. perfringens type A human gastrointestinal disease isolates to affect rabbit ileal loops. $\mathrm{Mol}$ Microbiol 33, 946-958.

Setlow, P. (2003). Spore germination. Curr Opin Microbiol 6, 550-556. Setlow, B., Peng, L., Loshon, C. A., Li, Y.-Q., Christie, G. \& Setlow, P. (2009). Characterization of the germination of Bacillus megaterium spores lacking enzymes that degrade the spore cortex. J Appl Microbiol 107, 318-328.

Shimamoto, S., Moriyama, R., Sugimoto, K., Miyata, S. \& Makino, S. (2001). Partial characterization of an enzyme fraction with protease activity which converts the spore peptidoglycan hydrolase (SleC) precursor to an active enzyme during germination of Clostridium perfringens S40 spores and analysis of a gene cluster involved in the activity. J Bacteriol 183, 3742-3751.

Shimizu, T., Ohtani, K., Hirakawa, H., Ohshima, K., Yamashita, A., Shiba, T., Ogasawara, N., Hattori, M., Kuhara, S. \& Hayashi, H. (2002). Complete genome sequence of Clostridium perfringens, an anaerobic flesh-eater. Proc Natl Acad Sci U S A 99, 996-1001.

Shinde, U. \& Inouye, M. (1996). Propeptide-mediated folding in subtilisin: the intramolecular chaperone concept. Adv Exp Med Biol 379, 147-154.

Siezen, R. J. \& Leunissen, J. A. (1997). Subtilases: the superfamily of subtilisin-like serine proteases. Protein Sci 6, 501-523.

Urakami, K., Miyata, S., Moriyama, R., Sugimoto, K. \& Makino, S. (1999). Germination-specific cortex-lytic enzymes from Clostridium perfringens S40 spores: time of synthesis, precursor structure and regulation of enzymatic activity. FEMS Microbiol Lett 173, 467-473.

Zhao, Y. \& Melville, S. B. (1998). Identification and characterization of sporulation-dependent promoters upstream of the enterotoxin gene (cpe) of Clostridium perfringens. J Bacteriol 180, 136-142.

Edited by: T. Abee 